\title{
mirror encodes a novel PBX-class homeoprotein that functions in the definition of the dorsal-ventral border in the Drosophila eye
}

\author{
Helen McNeill, ${ }^{1}$ Chung-Hui Yang, ${ }^{1}$ Michael Brodsky, ${ }^{2}$ Josette Ungos, ${ }^{1}$ and Michael A. Simon ${ }^{1,3}$ \\ ${ }^{1}$ Department of Biological Sciences, Stanford University, Stanford, California 94305 USA; ${ }^{2}$ Department of Biology, \\ Massachusetts Institute of Technology, Cambridge, Massachusetts 02139 USA
}

\begin{abstract}
The Drosophila eye is composed of dorsal and ventral mirror-image fields of opposite chiral forms of ommatidia. The boundary between these fields is known as the equator. We describe a novel gene, mirror (mrr), which is expressed in the dorsal half of the eye and plays a key role in forming the equator. Ectopic equators can be generated by juxtaposing mrr expressing and nonexpressing cells, and the path of the normal equator can be altered by changing the domain of mrr expression. These observations suggest that mrr is a key component in defining the dorsal-ventral boundary of tissue polarity in the eye. In addition, loss of mrr function leads to embryonic lethality and segmental defects, and its expression pattern suggests that it may also act to define segmental borders. Mirror is a member of the class of homeoproteins defined by the human proto-oncogene PBX1. mrr is similar to the Iroquois genes ara and caup and is located adjacent to them in this recently described homeotic cluster.
\end{abstract}

[Key Words: Drosophila; eye development; polarity; compartment; border]

Received January 14, 1997; revised version accepted March 4, 1997.

The spatial control of differentiation is a central issue in the study of the development of multicellular organisms. A primary step in pattern formation is the division of homogeneous cells into distinct fields. The border between these fields can then act as signaling centers for further patterning and growth (Peifer and Bejsovec 1992; Blair 1995). An example of such a border is found in the Drosophila eye. The eye is composed of dorsal and ventral fields of photoreceptor clusters called ommatidia. Ommatidia in the dorsal half of the eye are the mirror image of those in the ventral region (see Fig. 1B, below; Deitrich 1909; Ready et al. 1976; Tomlinson 1985, 1988; Tomlinson and Ready 1987). The boundary where the dorsal and ventral fields meet is known as the equator. The equator bisects the eye from anterior to posterior, rarely deviating by more than one ommatidial width as it crosses the eye. How the equator is determined is not well understood, but clonal analysis has indicated that cell lineage is not an important factor in forming the equator. Therefore, it has been proposed that cell-cell interactions are critical for establishing the dorsal/ventral (D/V) boundary within the eye (Ready et al. 1976).

The eye develops from a monolayer epithelium called the eye imaginal disc (for review, see Wolff and Ready

${ }^{3}$ Corresponding author.

E-MAIL msimon@leland.stanford.edu; FAX (415) 725-9832.
1993). During the third instar larval period preclusters at the posterior of the eye disc begin to form, and their differentiation induces the formation of new preclusters anterior to them. This leads to a wave of differentiation that sweeps across the disc. This wave is marked by an indentation known as the morphogenetic furrow, which separates the undifferentiated and differentiating regions of the disc. Initially, preclusters emerging from the morphogenetic furrow appear identical. As they mature, the preclusters in the dorsal and ventral regions of the eye rotate in opposite directions (see Fig. 1A, below). Asymmetries are incorporated into the preclusters as they rotate, resulting in ommatidia of opposing polarity and chirality in the adult eye. Recent work has demonstrated that the degree of ommatidial rotation is controlled by a number of genes including nemo and roulette /Choi and Benzer 1994) and has suggested that tissue polarity genes such as spinylegs, prickly-spinylegs (Gubb 1993; Choi et al. 1996), frizzled (Zheng et al. 1995), and dishevelled (Theisen et al. 1994) are necessary for regulating $\mathrm{D} / \mathrm{V}$ polarity in the eye.

Several experiments suggest that the equator might be source of $\mathrm{D} / \mathrm{V}$ patterning information. Analysis of patterning defects in clones of frizzled ${ }^{-}$cells suggests that frizzled is required to interpret and relay a signal emitted by the equator (Zheng et al. 1995). Consistent with this proposal is the observation that a number of genes ap- 
pear to be expressed either solely at the equator or in a gradient from the equator (Sun et al. 1995; Villano and Katz 1995; Brodsky and Steller 1996). Futhermore, morphological analysis has indicated that ommatidial differentiation in each row proceeds from the equator outwards (Wolff and Ready 1991) and has been used to suggest that more mature ommatidia provide $\mathrm{D} / \mathrm{V}$ polarity information to their less differentiated neighbors /Gubb 1993).

We describe here a novel homeodomain-containing protein, Mirror (Mrr), that plays an important role in defining the equator. $m r r$ is expressed in the dorsal half of the eye, with expression terminating at the equator. Using loss-of-function mrr alleles and ectopic expression of $m r r$, we have analyzed the role of mrr during eye development. Our data suggest that the juxtaposition of mrr expressing and nonexpressing cells plays an important role in defining the equator. In addition, analysis of the mrr expression pattern and the mrr loss-of-function phenotype in embryos suggests that mrr also acts in the definition of the segmental border.

\section{Results}

Identification of a gene expressed in the dorsal half of the eye

$m r r$ was first detected using a $\mathrm{P}$ element $\left(m r r^{P 1}\right)$ that carries white and lacZ and is inserted into the $69 \mathrm{D}$ region of the third chromosome. Strong white gene expression was restricted to cells dorsal to the equator (Fig. 1C,D). This expression pattern suggested that $m r r^{p 1}$
Figure 1. Identification of a gene expressed in the dorsal half of the eye. (A) Development of polarity and chirality in an eye imaginal disc. For this and all subsequent pictures, dorsal is up, anterior is to the left. Undifferentiated cells are sequentially recruited into the precluster. The aquisition of neuronal identity is indicated by the aquistion of color; R2/5 (purple), R3/4 (green), R8 (yellow), R7 (red). Preclusters begin to rotate as they differentiate, rotating in opposite directions above and below the equator. The final arrangement shown is not achieved until pupal stages. (B) Diagram of a wild-type equator. Above the equator ommatidia are organized such that the apex of the trapezoid formed by the ommatidium points up, whereas in the ventral half of the eye the mirror image form exists. Each black circle represents a rhabdomere projected by a photoreceptor into the middle of the ommatidium. (C) $\mathrm{mrr}^{p 1}$ flies express the white gene (which results in red pigmentation) in the dorsal half of the eye. $(D)$ Section of a $m r r^{P 1}$ eye. Note the restriction of white (seen as yellow pigment granules in sections) to the dorsal half of the eye. Expression of white does not cross the equator but terminates at variable positions in the dorsal ommatidia by the equator. Position of the equator is indicated by the sawtooth red line. $(E) \beta$-Galactosidase staining of a $\mathrm{mrr}^{p 1}$ eye imaginal disc. Staining is restricted to the dorsal half of the eye disc. Staining decreases after the furrow has passed. Note the patch of staining in the antennal portion of the disc. The white arrow indicates the position of the morphogenetic furrow; the black arrowhead indicates the presumed position of the equator. $(F)$ In situ hybridization of $m r r$ riboprobe to an eye imaginal disc. Transcript is restricted to the dorsal anterior half of the eye disc, with expression ceasing at the morphogenetic furrow. Arrows as in $E$. (G) $\beta$-Galactosidase staining of a $m r r^{P 1}$ embryo. Staining reflects a segmentally repeated expression pattern of this enhancer trap. $(H)$ In situ hybridization of $\mathrm{mrr}$ riboprobe to a wildtype stage 11 embyro. Segmentally repeated staining resembles staining of embryos carrying $\mathrm{mrr}^{p 1}$.

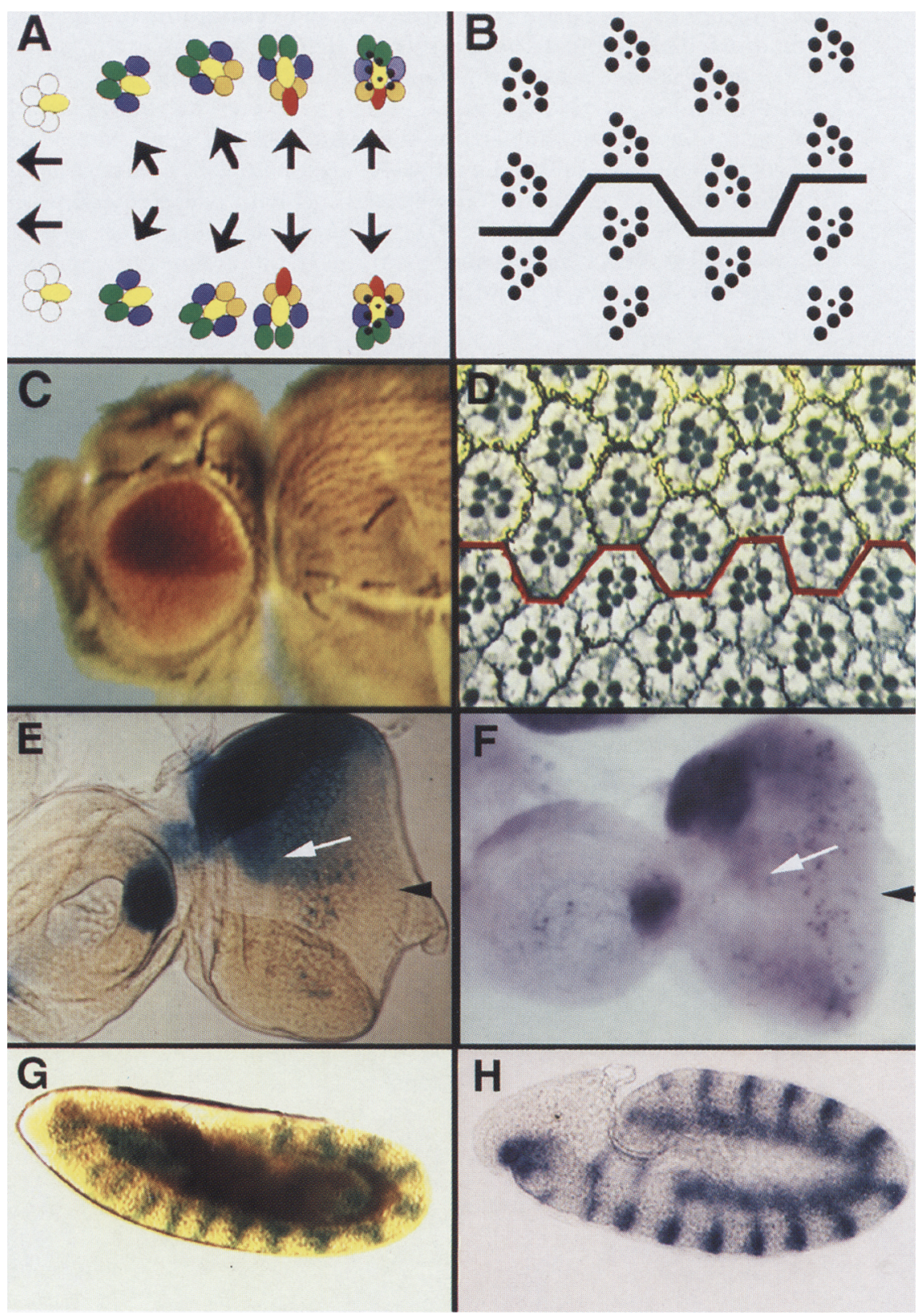


might be inserted close to a gene, that we call mrr, which is involved in determining dorsal identity or in forming the $\mathrm{D} / \mathrm{V}$ boundary. To determine if $\mathrm{mrr}$ is expressed at the time of the establishment of $\mathrm{D} / \mathrm{V}$ polarity, we examined developing eye discs. $\beta$-Galactosidase activity was restricted to the dorsal cells both in front of the morphogenetic furrow and in the first few rows behind the furrow (Fig. 1E). Analysis of $\beta$-galactosidase staining in $m r^{P 1}$ embryos (Fig. 1G) suggests that $m r r$ might also play a role in embryonic development.

\section{mrr encodes a homedomain protein}

To identify mrr, the P element was used to obtain genomic DNA adjacent to the insertion site. A chromosomal walk was conducted in both directions from the $m r r^{P 1}$ insertion point, and a portion of the walk was then used to probe an eye imaginal disc cDNA library (Fig. 2A). Nine overlapping cDNAs were isolated that define a 23$\mathrm{kb}$ transcription unit with five exons. Hybridization of these cDNAs to eye imaginal discs demonstrated that this gene is expressed in the dorsal half of the eye disc in a pattern similar to that of $\beta$-galactosidase expression in $m r r^{p 1}$ eye discs (Fig. 1E,F). Hybridization of these cDNAs to embryos also revealed expression that was very similar to $\beta$-galactosidase activity in $\mathrm{mrr}^{P 1}$ embryos (Fig. $1 \mathrm{G}, \mathrm{H})$. We conclude that this transcription unit is mrr.

The $3.5-\mathrm{kb}$ mrr transcript contains a single long open reading frame (ORF) of $1.9 \mathrm{~kb}$. Conceptual translation of the mrr cDNA (Fig. 2B) reveals that the mrr protein (Mrr) is a putative transcriptional activator containing both a homeodomain and an acidic transactivation domain (for review, see Triezenberg 1995; Burglin 1994). The Mrr homeodomain contains 3 additional amino acids be-
A

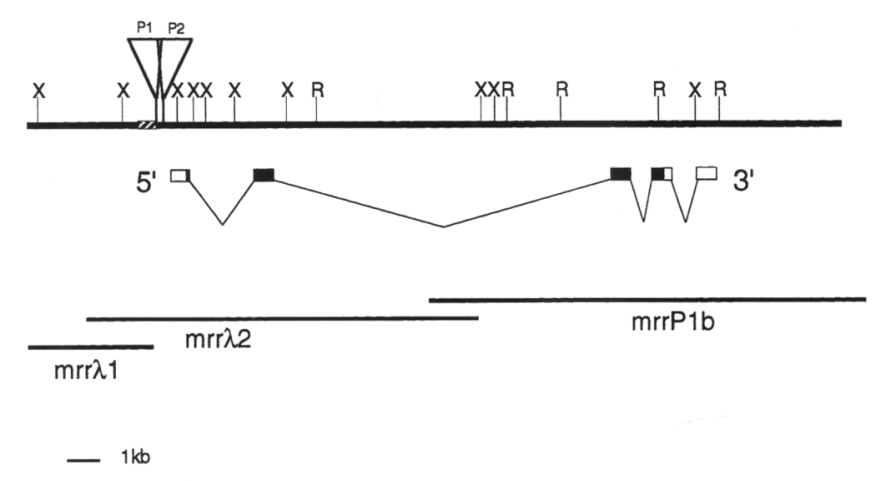

B ,

MTVHSNETGICLVMNAVSTHLATSSPNTPPTNAPQ SPPSTLPTLACPAAQPLSAPGIAGPGHSGMVTSTV PPGARSTS PCGPSAGL PALSLVGAPPPGPHSSAPG GAPAPGAGNPPNRCCDTGRTIYTDPVSGQTICSCQ YDMLNYQRLAAAGGVPLGVYPEGMSAYLSGIAADQ PPFYANPAGIDLKENLVAGASPWPYPSMYHPYDAA FAGYPFNSYGMDLNGARRKNATRETTSTLKAWLNE HKRNPYPTKGEK IMLA I ITKMTLTOVSTWFANARR

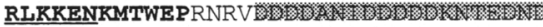
LLDAKDSGVGSTDDKDRSGRLGDMMTDRPGESNNS EWSESRPGSPNGSPDLYDRPGSMPPGAHPLFHPAA LHHHFRPPAGSPPDIAAYHHHQQQLLQQHQQAQQN SLQTAVGGTAKPR IWSLADMASKDSKDSSSGAKDN HPELPPAHPGFYGHPGQQPSPGKILSPLAARIPNY SPYVRTDLYRGFYGPAAAHLGAPTQEFLEHQRTFG ASLAAHNGPLGMNPLLWKAAVSGAANGPHFAPLSL TTSGGSSGGQQVAPPPVASPSASSSSSSMGCDVVH IPTSSGQSAAQHMMGPISSNSTASSSSSHSGKISP GVNVTSLSAKP

C

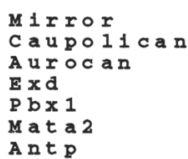

D

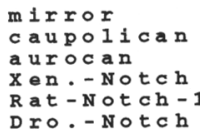

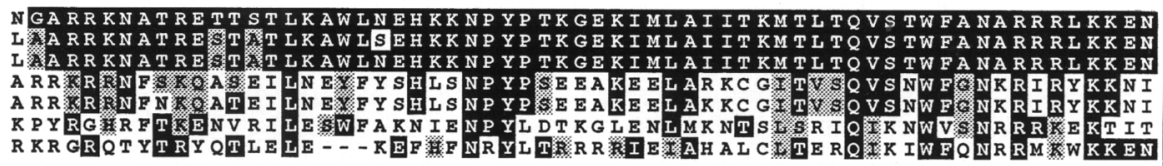

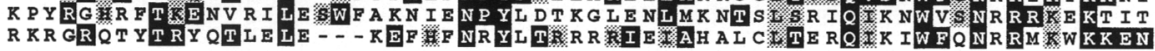

E

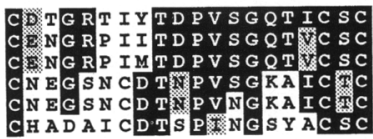

mirror

a uroc an

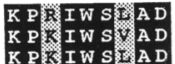

Figure 2. Molecular characterization of $m r r$. $(A)$ Genomic structure of the $m r r$ locus. A restriction map is shown. (X) Xho; (R) EcoRI; triangles indicate the positions of $\mathrm{P}$ elements determined by genomic sequencing. White boxes indicate the UTR of the $m r r$ cDNA; black boxes indicate coding regions. Positions of exons were determined through a combination of Southern hybridization and genomic sequencing of the intron-exon boundaries. $5^{\prime}$ and $3^{\prime}$ ends shown are those of the cDNA clones. Overlapping lines beneath the map indicate phage and $\mathrm{P} 1$ clones used to analyze the genomic region. The hatched region of the restriction map indicates the region excised in the embryonic lethal excision allele $m r r^{e 48}$, as determined by genomic Southern analysis of the mutant chromosome. $(B)$ Amino acid sequence of the Mrr protein deduced from the cDNA sequence. Coding begins with the first methionine of the ORF; however, the second methionine (downward arrows) conforms more closely to the Kozack consensus sequence. Bold characters indicate regions conserved with Ara and Caup. The underlined region indicates the position of the homeodomain. Shaded letters indicate acidic regions, which could act as transcriptional activatiors. (C) Homeodomain homology. Amino acids identical to Mrr are silhouetted in black; conservative changes are shaded. $(D)$ Notch interaction domain homology. (E) Novel homology region conserved between Mrr and Ara and Caup. 
tween helix 1 and helix 2 that are not present in most homeodomains. This places it in a class of homeodomain proteins defined by human PBX1 and its Drosophila homolog Extradenticle (see Fig. 2C) (Kamps et al. 1990; Nourse et al. 1990; Flegel et al. 1993; Rauskolb et al. 1993). These proteins are notable for forming heterodimers with other homeodomain proteins and increasing their partners' target specificity and affinity (Chan et al. 1994; van Dijk and Murre 1994, 1995; Chang et al. 1995; Knoepfler and Kamps 1995; Lu et al. 1995; Mann 1995; Phelan et al. 1995).

Mrr is related most closely to the two homeodomain proteins of the Iroquois complex, Araucan (Ara) and Caupolican (Caup), although the Iroquois proteins are related more closely to each other than to Mrr (GomezSkarmeta et al. 1996). The homology to these proteins is particularly striking in three regions (Fig. 2B). The major region of homology lies within the homeodomain and the 15 amino acids immediately after the homeodomain. Over the homeodomain, 57/60 amino acids are identical, with three conservative changes (Fig. 2C). In the aminoterminal region there is a highly conserved Notch interaction domain, which has been proposed to be involved in protein-protein interactions (Gomez-Skarmeta et al. 1996) (Fig. 2D). In addition, a highly conserved novel region close to the carboxyl terminus is also conserved among all these family members (Fig. 2E). The similarity of mrr to ara and caup and its coincident location at 69D (within $50 \mathrm{~kb}$ of caup; Gomez-Skarmeta et al. 1996) suggests that $m r r$ is a new member of the Iroquois complex.

\section{Patterning defects in mrr alleles}

To analyze the function of $m r r$ we sought to obtain mutant $m r r$ alleles. Because the $m r^{P 1}$ insertion site is within $300 \mathrm{bp}$ of the beginning of the $m r r$ transcriptional unit, the insertion of the $\mathrm{P}$ element might have disrupted mrr expression. We therefore began our phenotypic analysis by examining homozygous $m r r^{P 1}$ eyes to determine whether there were defects in patterning. $m r r^{p 1}$ eyes are smooth, with no apparent disruptions in the packing of the ommatidial facets. However, closer analysis revealed subtle patterning defects. In $-20 \%$ of $m r r^{P 1}$ eyes $(9 / 50)$, at least one ommatidium close to the equator is misspecified, having ventral chirality and polarity but residing in the dorsal field, or vice versa. Such mispecified ommatidia are not found in wild-type eyes 10/50; see also Franceschini and Kirchfeld 1971; Ready et al. 1976). In addition, $m r r^{P 1}$ eyes had defects in the path of the equator across the eye. In wild-type eyes, the equator moves up and down one ommatidial width as it traverses the eye and rarely if ever moves more than two ommatidial widths at one time. In contrast, $\sim 10 \%$ of $m r r^{P 1}$ eyes displayed an irregular equator. These irregularities were either three consecutive steps in a single direction or a single step of three ommatidial widths (data not shown). These defects in $m r r^{P 1}$ flies suggested that $m r r$ might have a role in defining the equator.

The weak and variable defects in $m r r^{P 1}$ eyes and the lack of a $m r r^{P 1}$ embryonic phenotype despite strong ex- pression of $m r r$ in the embryo (Fig. $1 \mathrm{H}$ ) suggested that the $m r r^{P 1}$ might retain substantial function. We therefore sought stronger mrr alleles. We took advantage of the fact that transposase-catalyzed excision of $\mathrm{P}$ elements is often imprecise and can result in the deletion of flanking sequences. Among 95 transposase-induced excision events, we isolated eight excision alleles that fail to complement each other and have either embryonic or early larval lethal phenotypes. Consistent with these alleles being defective for mrr function, in situ hybridization of embryos homozygous for $\mathrm{mrr}^{e 48}$, a strong allele that contains a $1-\mathrm{kb}$ deletion in the promoter region, had only very low levels of mirror transcript. We also obtained an independent insertion into the $m r r$ locus, $\mathrm{mrr}^{p 2}$ (Brodsky and Steller 1996). $\mathrm{mrr}^{p 2}$ is an insertion $234 \mathrm{bp}$ upstream of the $5^{\prime}$ end of the mrr cDNA and results in early larval lethality that cannot be complemented by $m r r^{e 48}$. This recessive lethal phenotype could be reverted at high frequency $(45 / 58$ excision events) upon excision of the $\mathrm{P}$ element, indicating that the $\mathrm{P}$ element insertion near mrr was the cause of the lethal phenotype. Taken together, these results indicate that the defects in each of these alleles are the result of reduced mrr expression.

\section{Loss of mrr expression can define a new equator}

Because strong $m r r$ alleles have an embryonic lethal phenotype, we studied mrr function in the eye by examining clones of cells homozygous for loss-of-function $\mathrm{mrr} \mathrm{mu}-$ tations. Clones of homozygous mutant cells were generated by mitotic recombination and lacked the white gene and therefore pigment granules. Loss of $m r r$ function had different effects in different parts of the eye. In the ventral half of the eye, there were no effects, consistent with our observation that mrr expression is restricted to the dorsal half of the eye. Dorsal clones also had no significant disturbances in ommatidial polarity or chirality within the clone. These results suggested that $m r r$ is not required for the implementation of dorsal identity (see Fig. 3B,C).

In contrast to the lack of defects within mrr-minus clones, we noted a dramatic alteration in patterning at the border of certain clones. Ectopic equators formed at the equatorial borders of dorsal anterior clones where mrr-minus tissue abutted mrr-expressing tissue (see Fig. 3B). The wild-type ommatidia just outside of the clone adopted ventral polarity and chirality. Ventral patterning extended for one to two ommatidial widths, then the tissue resumed normal dorsal patterning. The formation of ectopic equators where mrr-expressing and -nonexpressing cells meet suggests that the juxtaposition of $m r$-expressing and -nonexpressing cells serves to define the normal equator (Fig. 3A).

\section{Loss of mrr changes the path of the normal equator}

If the juxtaposition of mrr-expressing and -nonexpressing cells defines the normal equator, then loss of mrr function in dorsal cells along the equator should affect its 


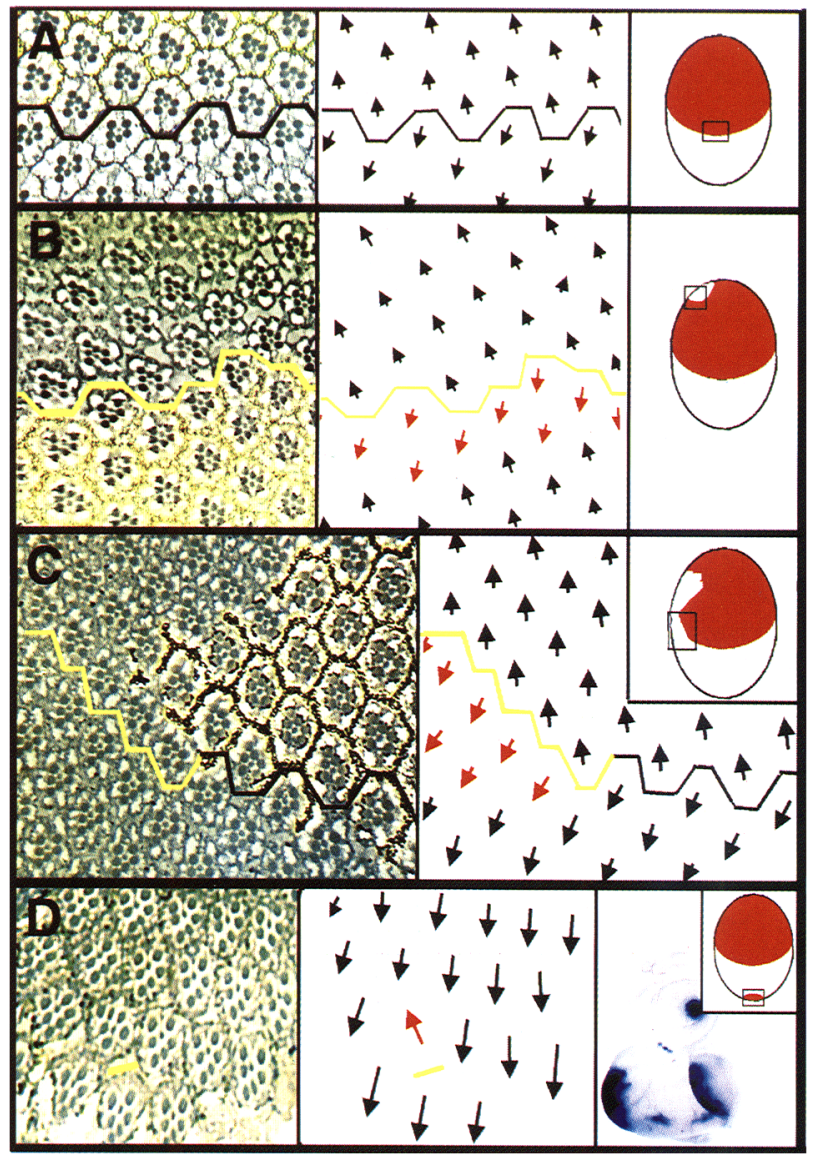

Figure 3. A border of mrr expression occurs at normal and at ectopic equators. Diagrams show approximate placement of sections: Red indicates mrr expression; white indicates lack of mrr expression. Aberrent or ectopic equators are indicated by a yellow line; a black line indicates a normal equator. Diagram of ommatidial polarity is adjacent. Black arrows indicate normal polarity; red arrows indicate abnormally oriented ommatidia. In sections $B$ and $C$ the loss-of-function clone is marked by the loss of yellow pigment granules. $(A)$ Section from a $m r r^{p 1}$ heterozygote eye, illustrating a border of $m r r$ expression at a wild-type equator. $(B)$ Ectopic equator generated at the ventral border of an anterior mrr-minus clone. All clones in the dorsal anterior region of the eye extended into head cuticle; therefore, analysis of the polar face of the clones was not possible. $(C)$ Equatorial clones demonstrating $m r r$ expression guiding the path of the equator as it enters a mrr-minus clone. $(D)$ Ectopic mrr expressed at the ventral margin of the eye can produce ectopic equators. The yellow line indicates the region of the ectopic equator. An eye imaginal disc from a UAS-1acZ; 30A Gal4 fly, stained for $\beta$-galactosidase activity to show the expression of the Gal4 line.

path. We therefore examined mrr-minus clones that crossed the normal equator. Where the equator approaches a patch of mrr-minus tissue, the path of the equator is diverted to follow the new border of $\mathrm{mrr}$ expression (Fig. 3C). This diversion is consistent with the proposal that the border of mrr expression is important for defining the position of the equator. However, after taking several dorsal steps paralleling the border of mrrexpressing and -nonexpressing cells, the equator resumes its posterior to anterior path across the eye rather than continuing to follow the edge of the clone. This result suggests that the pattern of mrr expression is not the sole determinant of equator position and that there may be spatial restrictions on the ability of $m r r$ to define the equator.

\section{Ectopic expression of mrr alters ommatidial patterning}

To further test the hypothesis that the pattern of $m r r$ expression defines the equator, we analyzed the effects of ectopic expression of mrr. Our first approach was to place $m r r$ under the control of a heat shock promoter and observe the effects of ubiquitous expression during third instar larval development. Heat shock-driven mrr disrupts normal development and results in flies with rough eyes (data not shown). Sections of these eyes revealed numerous defects, including randomized polarity and chirality of ommatidia. The pleiotropic nature of these defects are difficult to interpret but are consistent with a requirement for spatially restricted mrr expression. To test whether a border of $m r r$ expression is important for its function, we used the Gal4 system (Brand and Perrimon 1993) to express mrr at the ventral margin of the eye. Sections of these eyes revealed occasional ommatidia (in three of five eyes examined) that had dorsal polarity and chirality close to the ventral margin of the eye, near where ectopic mrr was expressed (Fig. 3D). No abnormal ommatidia were observed in regions of the ventral field away from where $m r r$ was ectopically expressed. These data suggest that ectopic expression of mrr can result in the perception of an equatorial border by nearby ommatidia and can lead them to orient around this new border.

\section{mrr expression during development}

Loss of mrr function results in embryonic lethality, which indicates that mrr function is important during early development. We therefore examined $m r r$ expression during embryogenesis by in situ hybridization. $\mathrm{mrr}$ expression was detected first at the cellular blastoderm stage as an anterior ventral patch at the site of the presumptive anterior midgut invagination. Shortly thereafter, $\mathrm{mrr}$ is expressed at the dorsal folds prior to their formation (Fig. 4A). mrr continues to be expressed in these tissues (Fig. 4B,C). mrr is expressed in a segmental pattern by the beginning of stage 10 (Fig. 4D) and then later in delaminating neuroblasts (Broadus et al. 1995). $m r r$ is also expressed at this time in more dorsal groups of cells. As the embryo undergoes germ-band retraction, mrr expression is retained in the nerve cord and appears transiently in the proventriculus as it undergoes folding (Fig. 4E).

mrr is also expressed in a complex pattern in larvae and adults. This pattern includes portions of the wing, haltere, and genital imaginal discs, as well as the dorsal anterior follicle cells in the ovary (data not shown). A 
Figure 4. In situ hybridization with riboprobes to staged embryos reveals dynamic expression of mrr during embryogenesis. Embryos are oriented so that dorsal is up and anterior is to the left. Embryos were staged according to Campos-Ortega and Hartenstein (1985). (A) Expression at dorsal folds and anterior midgut invagination; $|B|$ early gastrulation maintains pattern at dorsal folds and anterior midgut invagination; $(C)$ early germ-band extension maintains expression at dorsal folds and anterior midgut invagination; $(D)$ mrr is expressed in a segmentally repeated pattern in a germband extended, early stage $11 \mathrm{em}$ bryo; $(E)$ stage 13 embryos show strong staining in the proventriculus and weak staining in anterior portion of the ventral nerve cord; $(F)$ costaining with antibodies to engrailed (brown) and in situ $m r r$ probes (blue) demonstrate that $m r r$ is expressed at the anterior border of the segment.
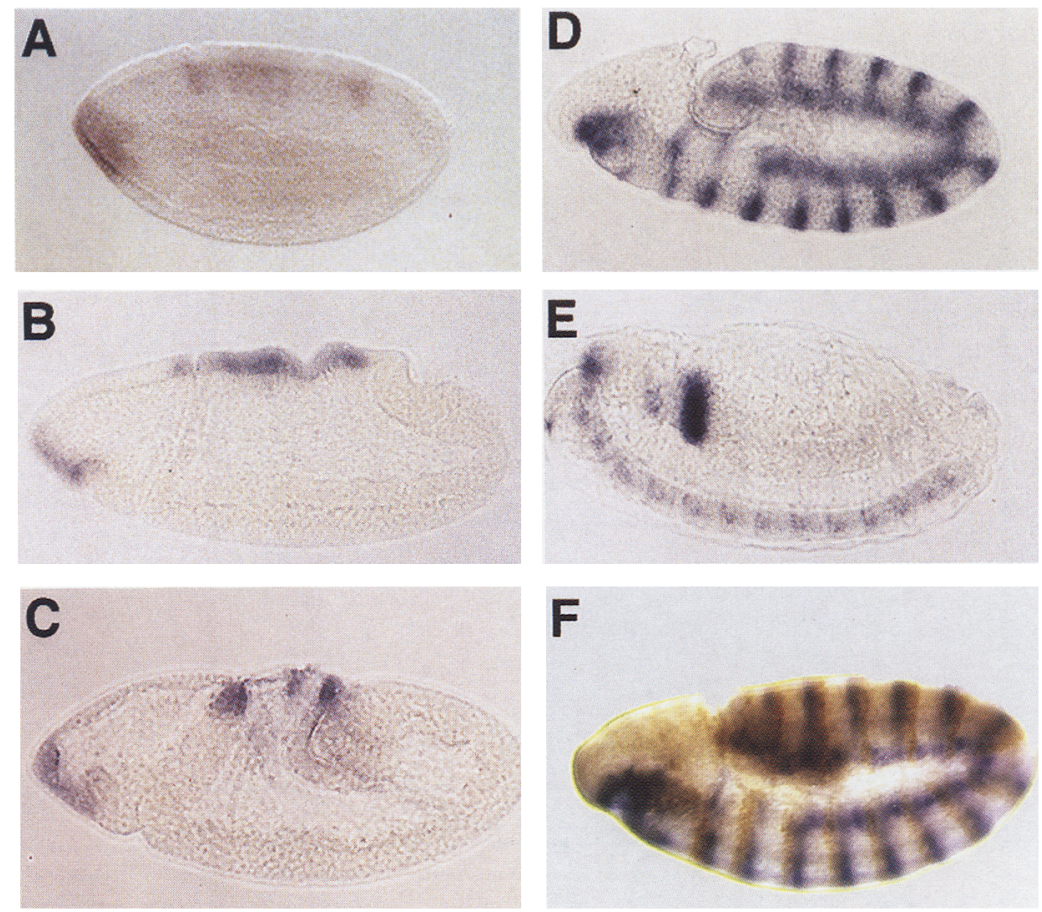

role for mrr function in the development of these tissues is reflected by defects in these structures in $m r^{P 1}$ flies. These defects include held-out wings, missing thoracic bristles, crumpled halters, and the production of eggs lacking dorsal appendages and anterior chorion.

\section{mrr function in embryonic segmentation}

The expression pattern of mrr suggests that its function may be important for many steps in development. We were particularly interested in the expression of $\mathrm{mrr}$ in each segment. To determine where in the segment $m r r$ is expressed, we costained wild-type embryos with antibodies to Engrailed, which defines the posterior border of each segment, and with mrr riboprobes. This experiment revealed that mrr expression is adjacent and posterior to Engrailed in every segment (Fig. 4F). This placed $\mathrm{mrr}$ expression at the anterior border of each segment.

To determine whether mrr expression in the segment is important for pattterning, we examined the cuticles of mrr loss-of-function embryos. mrr mutant cuticles were smaller than wild type and had sparse denticles (Fig. 5). Anterior denticles were often missing from each segment, and denticle rows 2 and 3 often were fused together. These defects in mrr mutant embryos confirm that mrr has a role in patterning within segments. Interestingly, a small percentage of embryos also had fusions of adjacent segments (Fig. 5E), suggesting that mrr may also play a role in defining the border between segments.

\section{Discussion}

The Drosophila eye is divided precisely into dorsal and ventral mirror-image fields, which are separated by a sharp boundary known as the equator. We have identified a novel homeobox-containing gene, mrr, which functions to define the equator. Three lines of evidence suggest that a border of mrr expression defines the equa-
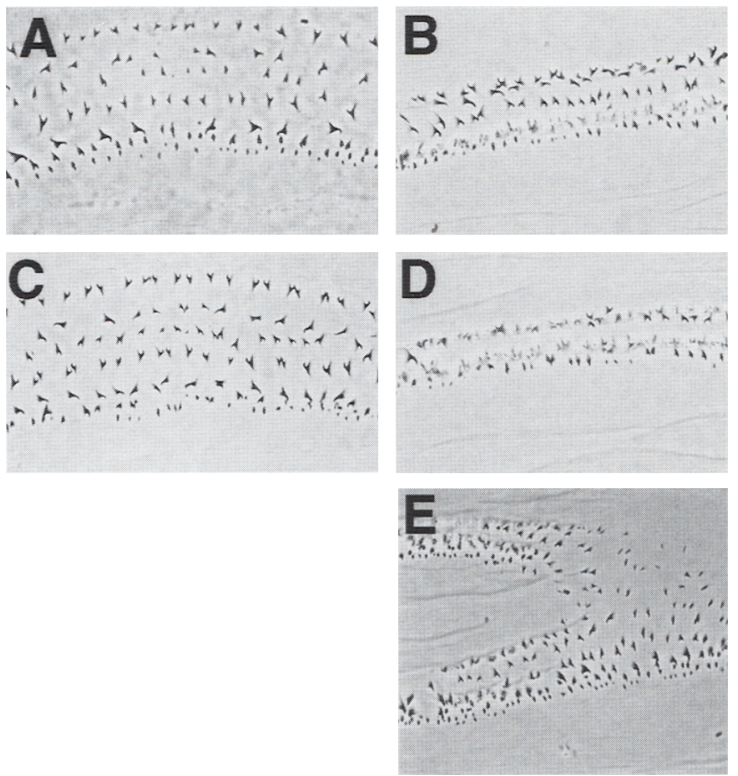

Figure 5. Loss of $m r r$ results in segmental defects. (A) Wildtype cuticle segments $A 5 ;(B)$ mrr mutant cuticle segment A5; demonstrating cuticle defects in homozygous mrr mutant embryos; $(C)$ wild-type cuticle segment A6; (D) mrr mutant cuticle segment $\mathrm{A} 6 ;(E)$ rare fusion event in mrr mutant embyro. Abdominal segment A4 and A5 have fused partially. Embryos were collected for $3 \mathrm{hr}$ and aged for $28 \mathrm{hr}$, dechorionated, devitellinized, and mounted in Hoyers/lactic acid. 
tor. First, the expression of $m r r$ is consistent with a role in defining the equator. $m r r$ is expressed in the dorsal half of the eye days before $\mathrm{D} / \mathrm{V}$ asymmetries become apparent, and expression ceases once these asymmetries are established. Furthermore, mrr expression terminates at the midpoint of the eye, producing a natural border of mrr expression where the equator will form. Second, our mosaic analysis has revealed that changes in mrr expression can create ectopic equators or alter the path of the normal equator. For example, mrr loss-of-function clones situated in dorsal regions of the eye can produce an ectopic equator along the side of the clone that faces the normal equator. In addition, when the normal equator enters a region of mrr loss of function, the path of the equator is diverted toward the new border of mrr expression. Third, ectopic expression studies are consistent with the hypothesis that a border of $m r r$ expression defines the equator. Ectopic expression of $m r r$ in a small region at the ventral margin of the eye results in the adoption of dorsal polarity and chirality of some nearby ommatidia. This suggests that an ectopic equator is being defined in this region. In contrast, ubiquitous expression of mrr led to rough and disordered eyes, which suggests that spatial restriction of mrr expression is important for the ability of mrr to define a border. Together, these data suggest a model in which the juxtapositioning of $m r$-expressing and -nonexpressing cells defines a border.

One important aspect of the induction of ectopic borders in mrr clones is that only the edge of the clone that is parallel to the normal equator produces an ectopic equator. One interpretation of this observation is that the polarizing signal is transmitted from an equator toward the dorsal and ventral poles. Consistent with this idea is the observation that clones of the tissue polarity mutant, frizzled, behave as though a signal is being transmitted from the equator (Zheng et al. 1995).

Although our results suggest that juxtaposing mrr-expressing and -nonexpressing cells can define a border, it is not always sufficient. Only clones in the anterior region form ectopic equators. All clones in the dorsal anterior-most region of the eye developed ectopic equators at their ventral face. One explanation for this restriction may be that $m r r$ is not the sole determinant of the equator. In this regard, it is interesting to note that ara and caup are also expressed in dorsal regions of the eye (J. Gomez-Skarmeta and J. Modellel, pers. comm.). In addition, there is evidence for the differential expression of other genes along the $\mathrm{D} / \mathrm{V}$ axis of the eye (Sun et al. 1995; Brodsky and Steller 1996). Such genes might contribute to equator formation. Alternatively, as our alleles are not entirely lacking $\mathrm{mrr}$ transcript, the possibility remains that clones without any mrr function could induce equators throughout the dorsal half of the eye.

\section{How is the equator formed?}

We propose that dorsal identity is determined through a gradient of a dorsal morphogen, which activates $\mathrm{mrr}$ transcription in a graded fashion. If $m r r$ is then able to regulate its own transcription (a characteristic of many transcription factors), this could result in a sharp border of $m r r$ expression. Interactions between the mrr-expressing and -nonexpressing cells could then lead to the production of signals that pattern the eye. Three observations are consistent with this model. First, a potential Mrr-binding site (based on the defined DNA binding site of Ara; Gomez-Skarmeta et al. 1996) is present in the mrr upstream region (H. McNeill, unpubl.). Second, mrr expression includes both a gradient that is strongest in the most dorsal regions and a sharp termination at the equator (see Fig. 1E,F). Third, there are several enhancer trap lines that are expressed at the equator, which provide candidates for control by Mrr.

Previous studies have been divided as to how D/V polarity and the equatorial boundary are established (Chanut and Heberlein 1995; Ma and Moses 1995; Strutt and Mlodzik 1995; Wehrli and Tomlinson 1995). Some groups suggest that $\mathrm{D} / \mathrm{V}$ polarity responds to global coordinates, whereas others propose that $\mathrm{D} / \mathrm{V}$ polarity results solely from progression of the morphogenetic furrow. The restriction of mrr expression to dorsal cells during the second larval instar clearly indicates that $\mathrm{D} / \mathrm{V}$ differences in positional identity exist prior to the furrow.

The mechanism of $\mathrm{D} / \mathrm{V}$ boundary determination in the wing may provide a model for understanding equator formation in the eye. Dorsal identity in the wing is controlled by homeodomain protein Apterous, which is expressed solely by dorsal cells (Bourgouin et al. 1992; Cohen et al. 1992; Williams et al. 1993). The juxtaposition of Apterous-expressing and -nonexpressing cells defines the $\mathrm{D} / \mathrm{V}$ border in part by directing dorsal expression of the secreted protein Fringe (Diaz and Cohen 1993; Blair et al. 1994; Irvine and Wieschaus 1994; Kim et al. 1995). Fringe then directs patterning at the $\mathrm{D} / \mathrm{V}$ border in part by controlling the Notch pathway (Kim et al. 1995, 1996). Because Apterous is not expressed in the eye, it is interesting to speculate that $\mathrm{mrr}$ may control D/V border formation in the eye by controlling some of these same genes. However, there are some differences in the actions of apterous and mrr. Although the equator can cross a clone of mrr cells, the $\mathrm{D} / \mathrm{V}$ border of the wing cannot cross through an apterous clone. Another difference is that cells within dorsal apterous clones have ventral identity while ommatidia within dorsal mrr clones always retain dorsal morphology. However, the lack of independent markers for $\mathrm{D} / \mathrm{V}$ identity in the eye prevents us from ruling out the possibility that the ommatidia within the dorsal mrr clones are actually ventral with respect to the ectopic equator.

\section{Mrr in the embryo}

Our study of mrr function in the eye has led us to propose that mrr acts to define the equatorial border. Our in situ analysis of $m r r$ expression demonstrated that $m r r$ is also expressed at another well-studied boundary, the segmental border. In this case, mrr is expressed at the anterior side of the border. The time of onset of mrr expres- 
sion in the segment is after the establishment of parasegmental signaling, but prior to and during the determination of the segmental border and the refinement of Engrailed and Wingless expression. As the border between Engrailed- and Wingless-expressing cells determines the parasegmental boundary, so might the juxtapositioning of mrr-expressing and -nonexpressing cells be important for defining the segmental border. Consistent with this hypothesis is our observation that $\mathrm{mrr}$ mutant embryos have segmental patterning defects. Our data in the $\mathrm{D} / \mathrm{V}$ boundary in the eye and on the segmental border in the embryo suggest that mrr may function in both of these systems to define borders.

\section{Materials and methods}

\section{Isolation and analysis of mrr mutations}

The $m r r^{p 1}$ insertion was obtained during a screen for imprecise excision of a P element in the Src64 locus, and was identified by the dorsal specific expression of the white gene. No Src64 interactions are observed. The P-element insertion site was localized to $69 \mathrm{D}$ on polytene chromosomes by in situ hybridization using biotin-labeled probes specific for P-element sequences and mrr cDNA probes.

mrr ${ }^{p 1}$ homozygous flies have numerous subtle defects. Flies have abnormal scutellar bristles, drooping halteres, dichaete wings, and are missing alulae. Homozygous mothers lay eggs lacking dorsal appendages and often have an open anterior chorion. Hatch rate analysis indicates that $<30 \%$ of eggs laid by homozygous mothers hatch. Reciprocal crosses to $w^{1118}$ stocks demonstrated that this is a maternal effect. Pseudopupil (Franceschini and Kirschfeld 1971) analysis of eyes indicated occasional subtle defects in the orientation of ommatidia and the regularity of the equator.

To obtain stronger $m r r$ alleles the $m r r^{P 1}$ insertion was mobilized using the $\Delta 2-3$ transposase source (Robertson 1988) and excisions were identified by loss of white expression.

\section{Immunohistochemistry and in situ analysis}

Digoxigenin-labeled mrr riboprobes were made using the Boehringer Manneheim Genius kit, as described. In situ hybridizations utilized antisense-strand riboprobes and were performed according to standard procedures (Tautz and Pfeifle 1989). Cuticle preparations in the eggshell were obtained by collecting the progeny of $m r r^{e 48} / w^{118}$ for $3 \mathrm{hr}$, aging embryos for $28 \mathrm{hr}$, devitellinizing, dechorianting, and mounting in Hoyers/lactic acid (Wieschaus and Nüsslein-Volhard 1986). Cuticle preparations were viewed after $24 \mathrm{hr}$ at $65^{\circ} \mathrm{C}$ on a slide warmer.

\section{Analysis of $\mathrm{mrr}^{\mathrm{P} 1} \beta$-galactosidase activity}

Imaginal discs were dissected from third instar $m r r^{p 1}$ homozygous larvae in phosphate-buffered saline (PBS), fixed in $1 \%$ glutaraldehyde for $5 \mathrm{~min}$ at $22^{\circ} \mathrm{C}$, and rinsed three times in PBS. Discs were immersed in X-gal staining solution for 1-3 hr at $22^{\circ} \mathrm{C}$. $\beta$-Galactosidase staining of $m r r^{P 1}$ and $m r r^{p 2}$ flies demonstrated that $m r r$ is expressed in wing imaginal discs in dorsal and peripheral regions, in ovaries in the anterior dorsal follicle cells, in genital disc in a lateral stripe, in the larval CNS, and intestinal tract. We did not observe staining in leg discs.

\section{Analysis of 30A Gal4: UAS mrr flies}

30A Gal4::UAS (upstream activating sequence) $m r r$ flies were obtained from a cross between $30 \mathrm{~A}$ Gal4/Cyo flies and UAS mir/TM3 flies. The resulting flies were raised at $18^{\circ} \mathrm{C}$ for 2 weeks and shifted to $23^{\circ} \mathrm{C}$ until eclosion. Flies carrying $\mathrm{mrr}$ expressed under the $30 \mathrm{~A}$ Gal4 control were identified by lack of TM3 and Cyo markers. Eyes from five flies were fixed with glutaraldehyde and osmium tetroxide as described (Tomlinson and Ready 1987) and analyzed in 1 um sections under a light microscope.

\section{Clonal analysis}

Mitotic clones were generated by irradiating the progeny of males (homozygous for a white ${ }^{+} \mathrm{P}$ element at $70^{\circ} \mathrm{C}$ ) crossed to $w^{-} ; m r r^{e 48} /$ TM3 females. Embryos were collected for $24 \mathrm{hr}$, aged for $24 \mathrm{hr}$, and irrradiated with 1000 rads. This generated $\mathrm{mrr}$ mutant clones marked by the loss of white. The positions of clones were marked prior to embedding, and standard histological methods were used for sections of adult eyes (Tomlinson and Ready 1987).

\section{Molecular biology}

Genomic DNA flanking $m r^{P 1}$ (a $1.5-\mathrm{kb}$ Xhol rescue fragment) was isolated by plasmid rescue (Pirrotta 1986) and used as a probe to screen a genomic library cloned into the $\lambda$ FixII vector (Stratagene, Inc). A 4.5-kb genomic fragment was then used to probe an eye imaginal disc cDNA library in $\lambda$ gt10 /constructed by Dr. A. Cowman, Walter and Eliza Hall Institute, Melbourne, Australia). EcoRI fragments of $\mathrm{mrr}$ cDNA were cloned into Bluescript (Stratagene, Inc.) and used for sequencing and production of riboprobes. Molecular analysis of $m r^{p 1}$ imprecise excisions was done using genomic Southern analysis. Additional genomic DNA was obtained by screening Pl cosmid libraries with a mrr cDNA. PlC was subcloned as a Sac-Cla fragment from P1446. Manipulations of DNA and RNA were done according to the protocols of Sambrook et al. (1989). Expression constructs containing mrr cDNA were made by fusing the two fragments of mrr cDNA obtained from EcoRI and HindIII subcloning of the $\lambda$ gt 10 cDNA library to a common HindIII site. The construct containing the $5^{\prime}$-untranslated region (UTR), the entire coding region of mrr and $0.7 \mathrm{~kb}$ of $3^{\prime}$ UTR were cloned into the KpnI site of $\mathrm{pHXK}$ and subcloned into the Not 1 site of hsCasPer. The same KpnI fragment was cloned into the KpnI site of the GalUAS vector pUAST (Brand and Perrimon 1993). Constructs were injected into $w^{1118}$ flies using standard methods (Rubin and Spradling 1982).

\section{Sequencing}

All DNA sequences were determined using the dideoxy chain termination method with a Sequenase kit (U.S. Biochemical). Templates for the determination of the 3505-bp mrr cDNA sequences were generated by sonication of plasmid DNA and insertion of the sonicated fragments into the vector M13mp10. The entire sequence of $m r r$ was determined on both strands. Compressions were eliminated using a combination of deazanucleotide and dITP nucleotides and analysis of templates on formamide-containing gels. The genomic structure of $m r r$ was determined through a combination of exonuclease-deletion of the $5.5-\mathrm{kb}$ plasmid rescue fragment of $m r r^{P 2}$ and primer-based sequencing of the remainder of $m r r$ using oligonucleotide primers on genomic subclone P1C. 


\section{Acknowledgments}

We thank Tom Kornberg for the generous gift of Engrailed antibodies, Hermann Steller for his support of M.B. for part of this work, Juan Modellel, Francoise Chanut, and Kathy Matthews and the Bloomington Stock Center for fly stocks, M. Scott for help with cuticle analysis, and members of the Simon laboratory, and Craig Smibert for comments on this manuscript. This research was supported by a postdoctoral fellowship to H.M. from the American Cancer Society. M.A.S. is a Markey scholar in Biomedical Science.

The publication costs of this article were defrayed in part by payment of page charges. This article must therefore be hereby marked "advertisement" in accordance with 18 USC section 1734 solely to indicate this fact.

\section{Note added in proof}

The sequence data described in this paper have been submitted to GenBank under accession no. U95021.

\section{References}

Blair, S.S. 1995. Compartments and appendage development in Drosophila. BioEssays 17: 299-309.

Blair, S.S., D.L. Brower, J.B. Thomas, and M. Zavortink. 1994. The role of apterous in the control of dorsoventral compartmentalization and PS integrin gene expression in the developing wing of Drosophila. Development 120: 1805-1815.

Brand, A.H. and N. Perrimon. 1993. Targeted gene expression as a means of altering cell fates and generating dominant phenotypes. Development 118: 401-415.

Broadus, J., J.B. Skeath, E. Spana, T. Bossing, G. Technau, and C.Q. Doe. 1995. New neuroblast markers and the origin of the $\mathrm{aCC} / \mathrm{pCC}$ neurons in the Drosophila central nervous system. Mech. Dev. 53: 393-402.

Brodsky, M.H. and H. Steller. 1996. Positional information along the dorsal-ventral axis of the Drosophila eye: Graded expression of the four-jointed gene. Dev. Biol. 173: 428-446.

Bourgouin, C., S. Lundgren, and J. Thomas. 1992. Apterous is a Drosophila LIM domain gene required for the development of a subset of embryonic muscles. Neuron 9: 549-561.

Burglin, T.R. 1994. A comprehensive classification of homeobox genes. In A guidebook for homeobox genes (ed. D. Duboule), Oxford University Press, Oxford, UK.

Campos-Ortega, J.A. and V. Hartenestein. 1985. The embryonic development of Drosophila melanogaster. Springer-Verlag, Berlin, Germany.

Chan, S.K., L. Jaffe, M. Capovilla, J. Botas, and R.S. Mann. 1994. The DNA binding specificity of Ultrabithorax is modulated by cooperative interactions with extradenticle, another homeoprotein. Cell 78: 603-615.

Chang, C.P., W.F. Shen, S. Rozenfeld, H.J. Lawrence, C. Largman, and M.L. Cleary. 1995. Pbx proteins display hexapeptide-dependent cooperative DNA binding with a subset of Hox proteins. Genes \& Dev. 9: 663-674.

Chanut, F. and U. Heberlein. 1995. Role of the morphogenetic furrow in establishing polarity in the Drosophila eye. Development 121: 4085-4094.

Choi, K.W. and S. Benzer. 1994. Rotation of photoreceptor clusters in the developing Drosophila eye requires the nemo gene. Cell 78: 125-136.

Choi, K.W., B. Mozer, and S. Benzer. 1996. Independent determination of symmetry and polarity in the Drosophila eye. Proc. Natl. Acad. Sci. 93: 5737-5741.

Cohen, B., M. McGuffin, C. Pfeifle, D. Segal, and S.M. Cohen.
1992. apterous, a gene required for imaginal disc development in Drosophila encodes a member of the LIM family of developmental regulatory proteins. Genes \& Dev. 6: 715729.

Diaz, B.F. and S.M. Cohen. 1993. Interaction between dorsal and ventral cells in the imaginal disc directs wing development in Drosophila. Cell 75: 741-752.

Dietrich, W. 1909. Die Facettenaugen der Dipteren Z. Wiss. Zool. 92: 465-539.

Flegel, W.A., A.W. Singson, J.S. Margolis, A.G. Bang, J.W. Posakony, and C. Murre. 1993. Dpbx, a new homeobox gene closely related to the human proto-oncogene pbx1 molecular structure and developmental expression. Mech. Dev. 41: 155-161.

Franceschini, N. and K. Kirchfeld. 1971. Etude optique in vivo des elements photorecepteurs dans l'oiel compose de Drosophila. Kybernetik 9: 159-182.

Gomez-Skarmeta, J.L., R.D. del Corral, E. de la Calle-Mustienes, M.D. Ferre, and J. Modolell. 1996. Araucan and caupolican, two members of the novel Iroquois complex, encode homeoproteins that control proneural and vein-forming genes. Cell 85: 95-105.

Gonzalez, C.S. and G. Morata. 1995. Control of Drosophila adult pattern by extradenticle. Development 121: $2117-$ 2125 .

Gubb, D. 1993. Genes controlling cellular polarity in Drosophila. (Suppl.) Development 269-277.

Irvine, K.D. and E. Wieschaus. 1994. fringe, a Boundary-specific signaling molecule, mediates interactions between dorsal and ventral cells during Drosophila wing development. Cell 79: 595-606.

Kampls, M.P., C. Murre, X.-h. Sun, and D. Baltimore. 1990. A new homeobox gene contributes the DNA binding domain of the $\mathrm{t}(1 ; 19)$ translocation protein in pre-B ALL. Cell 60:547-555.

Kim, J., K.D. Irvine, and S.B. Carroll. 1995. Cell recognition, signal induction, and symmetrical gene activation at the dorsal-ventral boundary of the developing Drosophila wing. Cell 82: 795-802.

Kim, J., A. Sebring, J. Esch, M. Kraus, K. Vorwerk, J. Magee, and S. Carroll. 1996. Integration of positional signals and regulation of wing formation and identity by Drosophila vestigal gene. Nature 382: 133-138.

Knoepfler, P.S. and M.P. Kamps. 1995. The pentapeptide motif of Hox proteins is required for cooperative DNA binding with Pbx1, physically contacts Pbx1, and enhances DNA binding by Pbx1. Mol. Cell. Biol. 15: 5811-5819.

Lu, Q., P.S. Knoepfler, J. Scheele, D.D. Wright, and M.P. Kamps. 1995. Both Pbxl and E2A-Pbxl bind the DNA motif ATCAATCAA cooperatively with the products of multiple murine Hox genes, some of which are themselves oncogenes. Mol. Cell. Biol. 15: 3786-3795.

Ma, C. and K. Moses. 1995. Wingless and patched are negative regulators of the morphogenetic furrow and can affect tissue polarity in the developing Drosophila compound eye. Development 121: 2279-2289.

Mann, R.S. 1995. The specificity of homeotic gene function. BioEssays 17: 855-863.

Nourse, J., J. Mellentin, N. Galili, J. Wilkinson, E. Stanbridge, S. Smith, and M. Cleary. 1990. Chromosomal translocation $t(1 ; 19)$ results in synthesis of a homeobox fusion mRNA that codes for a potential chimeric transcription factor. Cell 60: $535-555$.

Peifer, M. and A. Bejsovec. 1992. Knowing your neighbors: Cell interactions determine intrasegmental patterning in Drosophila. Trends Genet. 8: 243-249. 
Phelan, M.L., I. Rambaldi, and M.S. Featherstone. 1995. Cooperative interactions between HOX and PBX proteins mediated by a conserved peptide motif. Mol. Cell. Biol. 15: 39893997.

Pirrotta, V. 1986. Cloning Drosophila genes. In Drosophila, a practical approach (ed. D.B. Roberts), pp. 83-110. IRL Press, Oxford, UK.

Rauskolb, C., M. Peifer, and E. Wieschaus. 1993. extradenticle, a regulator of homeotic gene activity, is a homolog of the homeobox-containing human proto-oncogene pbxl. Cell 74: 1101-1112.

Sambrook, J., E.F. Fritsch, and T. Maniatis. 1989. Molecular cloning: A laboratory manual. Cold Spring Harbor Laboratory Press, Cold Spring Harbor, NY.

Ready, D.F., T.E. Hanson, and S. Benzer. 1976. Development of the Drosophila retina, a neurocrystalline lattice. Dev. Biol. 53: $217-240$.

Robertson, H.M. 1988. A stable genomic source of P element transposase in Drosophila melanogaster. Genetics 118: 461470.

Rubin, G.M. and A.C. Spradling. 1982. Genetic transformation of Drosophila with transposable element vectors. Science 218: 348-353.

Shen, W.F., C.P. Chang, S. Rozenfeld, G. Sauvageau, R.K. Humphries, M. Lu, H.J. Lawrence, M.L. Cleary, and C. Largman. 1996. Hox homeodomain proteins exhibit selective complex stabilities with Pbx and DNA. Nucleic Acids Res. 24: 898-906.

Strutt, D.I. and M. Mlodzik. 1995. Ommatidial polarity in the Drosophila eye is determined by the direction of furrow progression and local interactions. Development 121:42474256.

Sun, Y.H., C.J. Tsai, M.M. Green, J.L. Chao, C.T. Yu, T.J. Jaw, J.Y. Yeh, and V.N. Bolshakov. 1995. White as a reporter gene to detect transcriptional silencers specifying position-specific gene expression during Drosophila melanogaster eye development. Genetics 141: 1075-1086.

Tautz, D. and C. Pfeifle. 1989. A non-radioactive in situ hybridization method for the localization of specific RNAs in Drosophila embyros reveals translational control of the segmentation gene hunchback. Chromosoma 98: 81-85.

Theisen, H., J. Purcell, M. Bennett, D. Kansagara, A. Syed, and J.L. Marsh. 1994. dishevelled is required during wingless signaling to establish both cell polarity and cell identity. $D e$ velopment 120: 347-360.

Tomlinson, A. 1985. The cellular dynamics of pattern formation in the eye of Drosophila. J. Embryol. Exp. Morphol. 89: 313-331.

1988. Cellular interactions in the developing Drosophila eye. Development 104: 183-193.

Tomlinson, A. and D. Ready. 1987. Cell fate in the Drosophila ommatidium. Dev. Biol. 122: 264-275.

Triezenberg, S. 1995. Structure and function of transcriptional activation domains. Curr. Opin. Genet. Dev. 5: 190-196.

van Dijk, M.A. and C. Murre. 1994. extradenticle raises the DNA binding specificity of homeotic selector gene products. Cell 78: 617-624.

van Dijk, M., L.T. Peltenburg, and C. Murre. 1995. Hox gene products modulate the DNA binding activity of $\mathrm{Pbx} 1$ and Pbx2. Mech. Dev. 52: 99-108.

Villano, J.L. and F.N. Katz. 1995. four-jointed is required for intermediate growth in the proximal-distal axis in Drosophila. Development 121: 2767-2777.

Wehrli, M. and A. Tomlinson. 1995. Epithelial planar polarity in the developing Drosophila eye. Development 121:24512459 .
Weischaus, E. and C. Nüsslein-Volhard. 1986. Looking at embryos. In Drosophila, a practicle approach (ed. D.B. Roberts), pp. 199-227. IRL Press, Oxford, UK.

Williams, J.A., S.W. Paddock, and S.B. Carroll. 1993. Pattern formation in a secondary field: A hierarchy of regulatory genes subdivides the developing Drosophila wing disc into discrete subregions. Development 117: 571-584.

Wolff, T. and D.F. Ready. 1991. The beginning of pattern formation in the Drosophila compound eye: The morphogenetic furrow and the second mitotic wave. Development 113: $841-850$.

1993. Pattern formation in the Drosophila retina. In The Development of Drosophila melanogaster (ed. M. Bate and A. Martinez-Arias), Vol. 2, pp. 1277-1326. Cold Spring Harbor Laboratory Press, Cold Spring Harbor, NY.

Zheng, L., J. Zhang, and R.W. Carthew. 1995. frizzled regulates mirror-symmetric pattern formation in the Drosophila eye. Development 121: 3045-3055. 


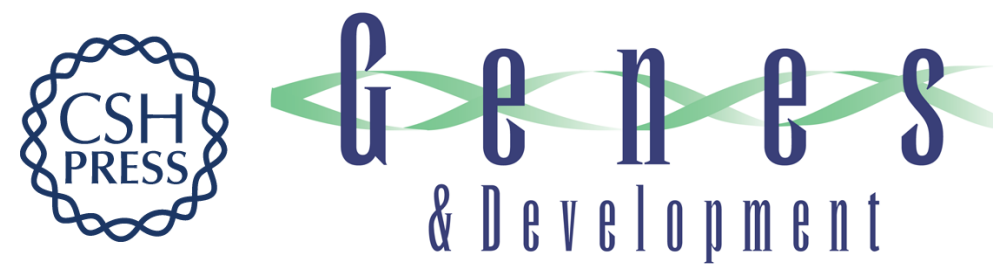

\section{mirror encodes a novel PBX-class homeoprotein that functions in the definition of the dorsal-ventral border in the Drosophila eye.}

$\mathrm{H}$ McNeill, C H Yang, M Brodsky, et al.

Genes Dev. 1997, 11:

Access the most recent version at doi:10.1101/gad.11.8.1073

References This article cites 49 articles, 21 of which can be accessed free at:

http://genesdev.cshlp.org/content/11/8/1073.full.html\#ref-list-1

License

Email Alerting

Service

Receive free email alerts when new articles cite this article - sign up in the box at the top right corner of the article or click here.

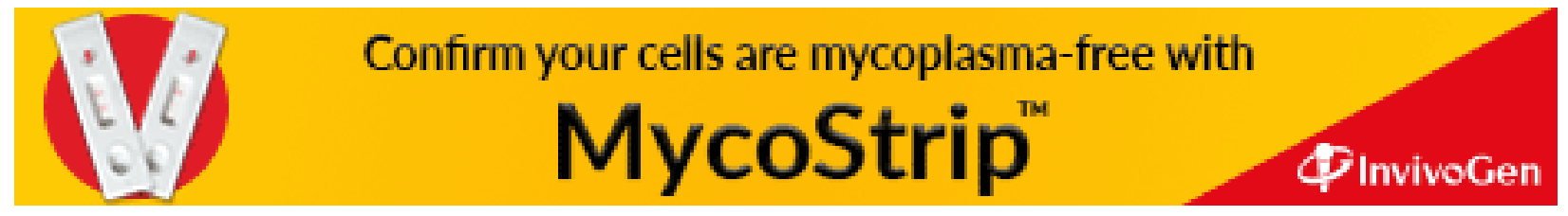

